\title{
Ressource et classification des eaux minérales et thermales en Allemagne
}

\author{
A. Herch et H. R. Langguth \\ RWTH Aachen, Allemagne
}

\section{INTRODUCTION}

En Allemagne* plus de 500 eaux minérales et thermales ont été reconnues dans les 10 dernières années selon la Directive de I'Union Européenne. Elles sont mises en bouteilles par un grand nombre des entreprises comme « eaux minérales naturelles ».

Les eaux de plus de 300 captages sont utilisées dans les stations thermales et balnéaires.

La diversité de ces eaux vient de la structure géologique complexe de l'Allemagne. La génèse des eaux minérales est étroitement liée à I'histoire géologique des différentes unités structurales.

CARLÉ (1975) a donné une description détaillée des "paysages d'eaux minérales "; une présentation sommaire avec une carte est due à FRICKE \& MICHEL (1969).

Il existe également des collections des analyses des eaux minérales régionales. Citons comme exemple DIENEMANN \& FRICKE (1961).

On peut distinguer 5 domaines principaux qui contiennent des provinces caractéristiques en eaux minérales et thermales :

- La Basse-Plaine au nord d'Allemagne. Il s'agit d'un grand bassin de sédimentation depuis le Permien avec une épaisseur de plusieurs milliers de mètres dans lequel se trouvent interstratifiés des dépôts salifères. La migration des sels dissous dans les eaux souterraines, la formation du pétrole et du gaz naturels, un diapirisme du sel ainsi que l'existence d'eaux connées des différentes périodes géologiques ont produit une vaste gamme de types d'eaux. Il y a beaucoup d'eaux salées et de saumures apparaissant à la surface ou trouvées dans des forages. L'existence du gypse est à l'origine des eaux sulfureuses et sulfurées. En bordure de la Plaine, les eaux salées pénètrent par des failles dans les massifs schisteux consolidés avoisinants.

- Massifs plissés. Les blocs plissés, le Massif Rhénan, le Hartz, la Forêt Noire et les complexes thuringo-franconiens et à l'est et au nord-est de la Bavière sont caractérisés par des eaux peu minéralisées. Proche du fossé Rhénan et en bordure des grands bassins sédimentaires et dans les zones qui subissent une lente ascension néotectonique combinée avec le volcanisme périalpin, des eaux carbogazeuses se sont formées, par exemple dans l’Eifel.

(*) Les analyses utilisées viennent presqu' exclusivement du territoire de l'ancienne République Fédérale Allemande.

\begin{abstract}
- Des grandes cuvettes sédimentaires mésozoïques (Triassique à Crétacé) renferment souvent des eaux typiques des différents niveaux géologiques. Les couches du Keuperien par exemple sont en général très riches en $\mathrm{SO}_{4}$. Mais aussi ces régions formant des montagnes moyennes comme la Hesse, le Palatinat, le Bade-Wurtemberg et la Franconie connaissent une activité néotectonique avec des manifestations volcaniques. Cela nous mène à des eaux gazeuses parfois mélangées avec des venues d'eau provenant des dépôts salifères intercalés.
\end{abstract}

- Le Fossé Rhénan qui est à cheval entre la France et l'Allemagne montre une richesse particulière en eaux thermominérales. Ce fossé a connu une subsidence depuis le Buntsandstein. Il a un potentiel hydrologique accentué entre les montagnes voisinantes. La formation du pétrole et du sel dans ce graben lui-même, des failles très importantes de plusieurs milliers de mètres de rejet ainsi qu'une mosaïque de blocs tectoniques à petite étendue sont à l'origine d'eaux d'une composition très différente du point de vue géochimique même situées très proches les unes des autres.

- Une province particulière se trouve dans le bassin molassique subalpin au sud du Danube jusqu'aux pieds des Alpes. Le remplissage des sédiments du Tertiaire comprenant des interstratifications saliferes et organiques (formation du pétrole et du gaz naturel) ainsi que le chevauchement des Alpes ont permis la formation d'eaux minérales à chimisme très varié. Une ressource aquifère particulière est celle des calcaires du Malmien dont les couches plongent régulièrement en direction SE du Jura Souabe jusqu'aux pieds des Alpes, c'est-à-dire de $+500 \mathrm{~m}$ à $-2000 \mathrm{~m}$ d'altitude. Les stations les plus récentes s'y trouvent, comme Bad Füssing, ainsi que des projets géothermiques intéressants.

\section{II — REPRÉSENTATIONS STATISTIQUES DE LA COMPOSITION GÉOCHIMIQUE}

Pour caractériser les eaux minérales en Allemagne nous avons choisi une représentation à l'aide des courbes cumulatives de fréquence relatives. L'axe des concentrations en $\mathrm{mg} / \mathrm{l}$ est toujours logarithmique.

Le tableau I résume d'abord les 35 paramètres chimiques avec leurs valeurs extrêmes, de l'ensemble de 680 analyses complètes qui étaient à notre disposition. Dans cette liste le nombre de données qui entrait dans le traitement statistique varie entre $678(\mathrm{Mg})$ et $52(\mathrm{Sb})$. Les 
Tableau 1. - Quantités d'éléments et de composants choisis et représentés sur les figures 2 à 6 avec leurs valeurs extrêmes.

\begin{tabular}{|c|c|c|c|c|}
\hline paramètre & nombre d'analyses & valeur minimale & valeur maximale & figure \\
\hline $\mathrm{Na}^{+}$ & 676 & 0,80 & 114300 & 2 \\
\hline $\mathrm{K}^{+}$ & 666 & 0,10 & 4875 & 2 \\
\hline $\mathrm{Ca}^{++}$ & 672 & 0,022 & 35900 & 2 \\
\hline $\mathrm{Mg}^{+*}$ & 678 & 0,20 & 3330 & 2 \\
\hline $\mathrm{Fe}^{++}$ & 615 & 0,0025 & 9830 & 3 \\
\hline $\mathrm{Mn}^{++}$ & 515 & 0,0003 & 420 & 5 \\
\hline & & & & \\
\hline $\mathrm{Cl}^{-}$ & 676 & 0,08 & 207500 & 2 \\
\hline $\mathrm{SO}_{4}{ }^{2-}$ & 666 & 0,20 & 11982 & 2 \\
\hline $\mathrm{HCO}_{3}{ }^{\circ}$ & 667 & 2,40 & 3671 & 2 \\
\hline $\mathrm{NO}_{3}^{-}$ & 371 & 0,002 & 298 & 5 \\
\hline & & & & \\
\hline $\mathbf{F}$ & 302 & 0,001 & 20 & 3 \\
\hline 5 & 293 & 0,0001 & 317 & 3 \\
\hline $\mathrm{Br}^{-}$ & 365 & 0,001 & 1360 & 4 \\
\hline $\mathrm{S}^{2-}$ & 85 & 0,0015 & 376,8 & 3 \\
\hline & & & & \\
\hline $\mathrm{CO}_{2}$ & 610 & 1,52 & 4774 & 3 \\
\hline $\mathrm{H}_{2} \mathrm{SiO}_{3}$ & 616 & 0,10 & 388 & 4 \\
\hline $\mathrm{H}_{2} \mathrm{TiO}_{3}$ & 128 & 0,0007 & 10,78 & 4 \\
\hline $\mathrm{HBO}_{2}$ & 227 & 0,007 & 280 & 5 \\
\hline & & & & \\
\hline$\overline{\mathrm{Ag}}$ & 89 & 0,00003 & 0,4 & 6 \\
\hline Al & 298 & 0,0005 & 1200 & 4 \\
\hline As & 93 & 0,00025 & 42,63 & 5 \\
\hline$\overline{\mathrm{Be}}$ & 55 & 0,00008 & 0,01 & 6 \\
\hline Co & 131 & 0,0002 & 2,7 & 4 \\
\hline $\mathrm{Cr}$ & 99 & 0,0001 & 1,5 & 5 \\
\hline Cs & 140 & 0,00004 & 10,75 & 6 \\
\hline $\mathrm{Cu}$ & 275 & 0,0002 & 1153 & 6 \\
\hline $\mathrm{Hg}$ & 61 & 0,00002 & 0,5 & 6 \\
\hline $\mathrm{Li}$ & 397 & 0,0016 & 163. & 6 \\
\hline$\overline{\mathrm{Ni}}$ & 193 & 0,0005 & 6,6 & 4 \\
\hline $\mathrm{Pb}$ & 208 & 0,0003 & 34 & 4,5 \\
\hline $\mathrm{Rb}$ & 106 & 0,0014 & 9,37 & 6 \\
\hline $\mathrm{Sb}$ & 52 & 0,00025 & 0,12 & 5 \\
\hline $\mathrm{Se}$ & 34 & 0,00005 & 0,2 & 5 \\
\hline $\mathrm{Sr}$ & 439 & 0,01 & 2200 & 6 \\
\hline$Z n$ & 286 & 0,0003 & 120 & 6 \\
\hline
\end{tabular}

concentrations maximales individuelles varient entre $207500 \mathrm{mg} / \mathrm{l}(\mathrm{Cl})$ et $0,01 \mathrm{mg} / \mathrm{l}(\mathrm{Be})$, les minimales entre $2,4 \mathrm{mg} / \mathrm{l}\left(\mathrm{HCO}_{3}\right)$ et $0,00002 \mathrm{mg} / \mathrm{l}(\mathrm{Hg})$.

La figure $I$ contient les sommes des éléments dissous en milligrammes par litre (ppm) et en milli-équivalents (epm). Les plus faibles concentrations en ppm sont autour de $130 \mathrm{mg} / \mathrm{l}$. Environ $17 \%$ des eaux ont des concentrations en dessous d'un gramme par litre. Ce sont des eaux faiblement minéralisées et actuellement très recherchées. Pour être reconnues comme eau minérale naturelle elles devraient passer un examen de l'efficacité de leurs propriétés physiothérapeutiques.

La plus haute concentration est celle d'une saumure au point de saturation en $\mathrm{NaCl}$.

La figure 2 donne la distribution des composants majeurs qui servent à la classification (voir tabl. 2 et $\S 4$ ). Il est bien visible que le sodium et le calcium ainsi que le $\mathrm{HCO}_{3}$ et 


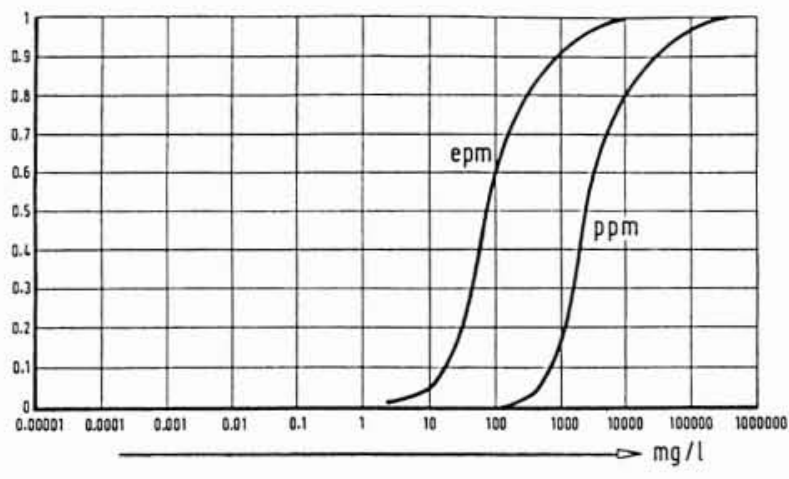

1. Distributions des concentrations en $\mathrm{mg} / \mathrm{l}$ et méq/l.

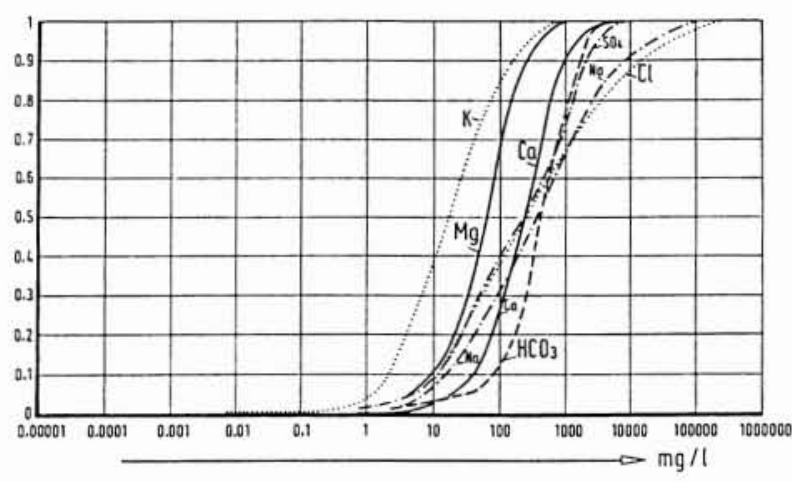

2. Distribution des éléments et des composants majeurs.

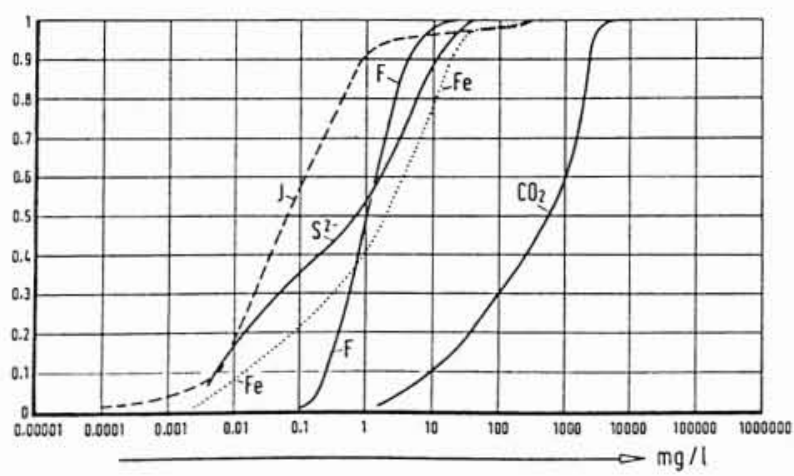

3. Distribution des éléments et des composants spécifiques.

le chlorure devraient apparaître le plus souvent dans une dénomination géochimique.

La figure 3 contient les composants mineurs qui peuvent apparaître dans l'appellation balnéaire comme élément spécifique lorsqu'ils atteignent et dépassent les teneurs suivantes :

$\begin{array}{lcc}\text { élément } & m g / l & \text { nombre dianalyses } \\ \mathrm{Fe} & 1 & 615 \\ \mathrm{I} & 1 & 293 \\ \mathrm{~S} & 1 & 111 \\ \mathrm{CO}_{2} & 1000 & 610 \\ \mathrm{~F} & 1 & 302\end{array}$

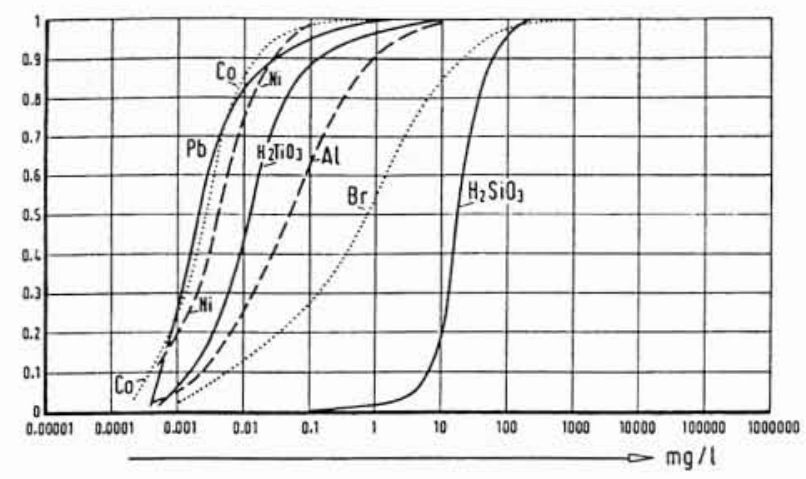

4. Distribution de quelques éléments et composants de trace.

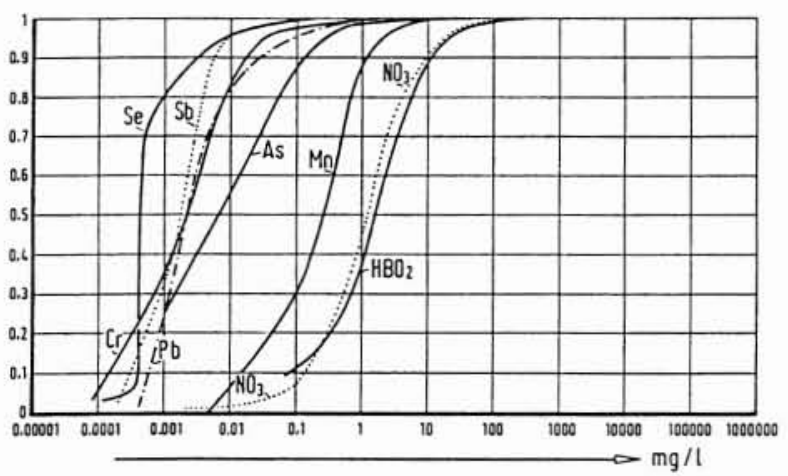

5. Distribution de quelques éléments et composants de trace.

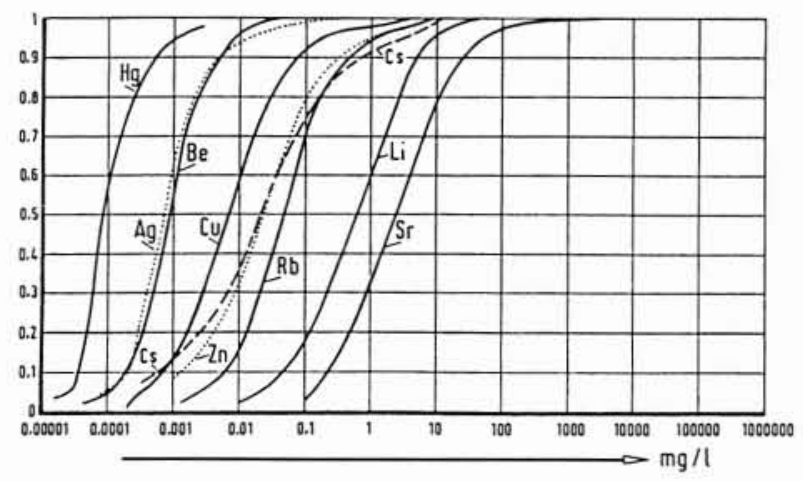

6. Distribution de quelques éléments et composants de trace.

Il est évident que $40 \%$ des eaux minérales sont des eaux naturellement carbogazeuses, c'est-à-dire $36 \%$ de l'ensemble d'échantillons.

Dans le cas de l'iode il n'y a que $10 \%$ d'environ la moitié de tous les échantillons qui dépassent la teneur exigée.

Les figures 4. 5 et 6 donnent un choix des éléments de trace. Par exemple les valeurs de As dépassent le seuil de $0,05 \mathrm{mg} / \mathrm{l}$ qui est la limite actuelle pour les eaux potables. 
Pour une mise en bouteille et pour la « buvette » dans les stations thermales il est donc nécessaire d'enlever l'arsenic.

Prenons comme deuxième exemple le nitrate : parmi les 371 échantillons, seulement $10 \%$ dépassent les $10 \mathrm{mg} / \mathrm{l}$, mais seulement $3 \%$, la limite pour les eaux potables.

\section{III $\square$ LES ANALYSES DANS LES DIAGRAM- MES PIPER}

Ces représentations relatives sont rangées en fonction de la concentration totale en $\mathrm{ppm}$ croissante $(=$ sumppm).

Figure 7: sumppm < $1000 \mathrm{ppm}$

Tableau 2. - Résumé de la classification géochimique pour les eaux minérales ayant une concentration totale entre 1000 et $10000 \mathrm{mg} / \mathrm{l}$.

\begin{tabular}{|c|c|c|c|c|}
\hline \multirow[t]{2}{*}{ Classification } & \multicolumn{2}{|c|}{ Nombre des eaux minerales par groupe } & \multicolumn{2}{|c|}{ Concentration [mg/l]] } \\
\hline & total & avec CO2 > $1000 \mathrm{mg} / \mathrm{l}$ & minimale & maximale \\
\hline \multicolumn{5}{|l|}{$\mathrm{HCO}_{3}$} \\
\hline $\mathrm{Ca}-\mathrm{HCO}_{3}$ & 8 & 7 & 1046 & 2096 \\
\hline $\mathrm{Ca}-\mathrm{Mg}-\mathrm{HCO}_{3}$ & 19 & 17 & 1103 & 3291 \\
\hline $\mathrm{Ca}-\mathrm{Mg}-\mathrm{Na}-\mathrm{HCO}_{3}$ & 3 & 2 & 2522 & 3395 \\
\hline $\mathrm{Ca}-\mathrm{Na}-\mathrm{HCO}_{3}$ & 8 & 7 & 1031 & 3521 \\
\hline $\mathrm{Ca}-\mathrm{Na}-\mathrm{Mg}-\mathrm{HCO}_{3}$ & 2 & 2 & 1224 & 1468 \\
\hline $\mathrm{Mg}-\mathrm{Ca}-\mathrm{HCO}_{3}$ & 3 & 3 & 1030 & 2293 \\
\hline $\mathrm{Na}-\mathrm{HCO}_{3}$ & 12 & 5 & 1314 & 9919 \\
\hline $\mathrm{Na}-\mathrm{Ca}-\mathrm{HCO}_{3}$ & 2 & 2 & 1722 & 2911 \\
\hline $\mathrm{Na}-\mathrm{Ca}-\mathrm{Mg}-\mathrm{HCO}_{3}$ & 5 & 4 & 1004 & 2756 \\
\hline $\mathrm{Na}-\mathrm{Mg}-\mathrm{HCO}_{3}$ & 8 & 8 & 1942 & 4973 \\
\hline $\mathrm{Na}-\mathrm{Mg}-\mathrm{Ca}-\mathrm{HCO}_{3}$ & 1 & 1 & 2288 & \\
\hline $\mathrm{Na}-\mathrm{HCO}_{3}-\mathrm{Cl}$ & 29 & 12 & 1122 & 6204 \\
\hline $\mathrm{Na}-\mathrm{Ca}-\mathrm{HCO}_{3}-\mathrm{Cl}$ & 1 & - & 1015 & \\
\hline $\mathrm{Na}-\mathrm{Ca}-\mathrm{Mg}-\mathrm{HCO}_{3}-\mathrm{Cl}$ & 1 & - & 1186 & \\
\hline $\mathrm{Na}-\mathrm{Mg}-\mathrm{HCO}_{3}-\mathrm{Cl}$ & 8 & 6 & 1013 & 7439 \\
\hline $\mathrm{Na}-\mathrm{Mg}-\mathrm{Ca}-\mathrm{HCO}_{3}-\mathrm{Cl}$ & 1 & 1 & 5033 & \\
\hline $\mathrm{Na}-\mathrm{HCO}_{3}-\mathrm{Cl}-\mathrm{SO}_{4}$ & 3 & 2 & 1343 & 3172 \\
\hline $\mathrm{Na}-\mathrm{Ca}-\mathrm{HCO}_{3}-\mathrm{Cl}-\mathrm{SO}_{4}$ & 1 & 1 & 2730 & \\
\hline $\mathrm{Na}-\mathrm{Ca}-\mathrm{Mg}-\mathrm{HCO}_{3}-\mathrm{Cl}-\mathrm{SO}_{4}$ & 2 & 2 & 3736 & 4356 \\
\hline $\mathrm{Ca}-\mathrm{Mg}-\mathrm{HCO}_{3}-\mathrm{SO}_{4}-\mathrm{Cl}$ & 1 & - & 1623 & \\
\hline $\mathrm{Ca}-\mathrm{Na}-\mathrm{HCO}_{3}-\mathrm{SO}_{4}-\mathrm{Cl}$ & 2 & - & 1460 & 1536 \\
\hline $\mathrm{Ca}-\mathrm{Na}-\mathrm{Mg}-\mathrm{HCO}_{3}-\mathrm{SO}_{4}-\mathrm{Cl}$ & 2 & - & 1717 & 1827 \\
\hline $\mathrm{Na}-\mathrm{Ca}-\mathrm{HCO}_{3}-\mathrm{SO}_{4}-\mathrm{Cl}$ & 2 & - & 1250 & 1338 \\
\hline $\mathrm{Ca}-\mathrm{HCO}_{3}-\mathrm{SO}_{4}$ & 4 & 3 & 1063 & 2912 \\
\hline $\mathrm{Ca}-\mathrm{Mg}-\mathrm{HCO}_{3}-\mathrm{SO}_{4}$ & 9 & 6 & 1010 & 2531 \\
\hline $\mathrm{Ca}-\mathrm{Mg}-\mathrm{Na}-\mathrm{HCO}_{3}-\mathrm{SO}_{4}$ & 2 & - & 1438 & 1793 \\
\hline $\mathrm{Ca}-\mathrm{Na}-\mathrm{HCO}_{3}-\mathrm{SO}_{4}$ & 5 & 2 & 1160 & 2862 \\
\hline $\mathrm{Ca}-\mathrm{Na}-\mathrm{Mg}-\mathrm{HCO}_{3}-\mathrm{SO}_{4}$ & 1 & 1 & 4460 & \\
\hline $\mathrm{Mg}-\mathrm{Ca}-\mathrm{HCO}_{3}-\mathrm{SO}_{4}$ & 1 & - & 1354 & \\
\hline $\mathrm{Na}-\mathrm{HCO}_{3}-\mathrm{SO}_{4}$ & 2 & - & 1098 & 2438 \\
\hline $\mathrm{Na}-\mathrm{Ca}-\mathrm{HCO}_{3}-\mathrm{SO}_{4}$ & 1 & 1 & 1397 & \\
\hline $\mathrm{Na}-\mathrm{Ca}-\mathrm{Mg}-\mathrm{HCO}_{3}-\mathrm{SO}_{4}$ & 1 & 1 & 1953 & \\
\hline $\mathrm{Na}-\mathrm{Mg}-\mathrm{HCO}_{3}-\mathrm{SO}_{4}$ & 2 & 2 & 1388 & 4659 \\
\hline
\end{tabular}




\begin{tabular}{|c|c|c|c|c|}
\hline \multirow[t]{2}{*}{ Classification } & \multicolumn{2}{|c|}{ Nombre des eaux minerales par groupe } & \multicolumn{2}{|c|}{ Concentration [mg/l] } \\
\hline & total & avec $\mathrm{CO} 2>1000 \mathrm{mg} / \mathrm{l}$ & minimale & maximale \\
\hline \multicolumn{5}{|l|}{$\mathrm{SO}_{4}$} \\
\hline $\mathrm{Ca}-\mathrm{SO}_{4}$ & 34 & - & 1676 & 2952 \\
\hline $\mathrm{Ca}-\mathrm{Mg}-\mathrm{SO}_{4}$ & 8 & 1 & 1381 & 3136 \\
\hline $\mathrm{Ca}-\mathrm{Mg}-\mathrm{Na}-\mathrm{SO}_{4}$ & 3 & 2 & 1339 & 2550 \\
\hline $\mathrm{Ca}-\mathrm{Na}-\mathrm{SO}_{4}$ & 5 & 2 & 1850 & 3674 \\
\hline $\mathrm{Ca}-\mathrm{SO}_{4}-\mathrm{HCO}_{3}$ & 18 & 5 & 1035 & 4721 \\
\hline $\mathrm{Ca}-\mathrm{Mg}-\mathrm{SO}_{4}-\mathrm{HCO}_{3}$ & 13 & 5 & 1093 & 4042 \\
\hline $\mathrm{Ca}-\mathrm{Mg}-\mathrm{Na}-\mathrm{SO}_{4}-\mathrm{HCO}_{3}$ & 2 & 1 & 2058 & 3610 \\
\hline $\mathrm{Ca}-\mathrm{Na}-\mathrm{SO}_{4}-\mathrm{HCO}_{3}$ & 9 & 4 & 1264 & 4643 \\
\hline $\mathrm{Ca}-\mathrm{Na}-\mathrm{Mg}-\mathrm{SO}_{4}-\mathrm{HCO}_{3}$ & 1 & - & 1768 & \\
\hline $\mathrm{Na}-\mathrm{Ca}-\mathrm{SO}_{4}-\mathrm{HCO}_{3}$ & 2 & - & 1406 & 2033 \\
\hline $\mathrm{Na}-\mathrm{Ca}-\mathrm{Mg}-\mathrm{SO}_{4}-\mathrm{HCO}_{3}$ & 1 & - & 2045 & \\
\hline $\mathrm{Na}-\mathrm{SO}_{4}-\mathrm{HCO}_{3}$ & 1 & - & 2038 & \\
\hline $\mathrm{Ca}-\mathrm{SO}_{4}-\mathrm{HCO}_{3}-\mathrm{Cl}$ & 2 & - & 1295 & 1496 \\
\hline $\mathrm{Ca}-\mathrm{Na}-\mathrm{SO}_{4}-\mathrm{HCO}{ }_{3}-\mathrm{Cl}$ & 3 & 1 & 1767 & 5045 \\
\hline $\mathrm{Mg}-\mathrm{Ca}-\mathrm{SO}_{4}-\mathrm{HCO}{ }_{3}-\mathrm{Cl}$ & 1 & - & 2797 & \\
\hline $\mathrm{Na}-\mathrm{Ca}-\mathrm{SO}_{4}-\mathrm{HCO}_{3}-\mathrm{Cl}$ & 1 & - & 1805 & \\
\hline $\mathrm{Ca}-\mathrm{SO}_{4}-\mathrm{Cl}-\mathrm{HCO}_{3}$ & 1 & - & 1162 & \\
\hline $\mathrm{Na}-\mathrm{Ca}-\mathrm{SO}_{4}-\mathrm{Cl}-\mathrm{HCO}_{3}$ & 2 & - & 1883 & 3847 \\
\hline & & & & \\
\hline $\mathrm{Ca}-\mathrm{SO}_{4}-\mathrm{Cl}$ & 2 & 1 & 1991 & 2762 \\
\hline $\mathrm{Ca}-\mathrm{Mg}-\mathrm{Na}-\mathrm{SO}_{4}-\mathrm{Cl}$ & 1 & 1 & 2440 & \\
\hline $\mathrm{Ca}-\mathrm{Na}-\mathrm{SO}_{4}-\mathrm{Cl}$ & 7 & - & 1765 & 4230 \\
\hline $\mathrm{Ca}-\mathrm{Na}-\mathrm{Mg}-\mathrm{SO}_{4}-\mathrm{Cl}$ & 2 & - & 1867 & 2718 \\
\hline $\mathrm{Na}-\mathrm{Ca}-\mathrm{SO}_{4}-\mathrm{Cl}$ & 3 & - & 5236 & 7654 \\
\hline $\mathrm{Na}-\mathrm{Mg}-\mathrm{Ca}-\mathrm{SO}_{4}-\mathrm{Cl}$ & 1 & - & 6346 & \\
\hline \multicolumn{5}{|l|}{$\mathrm{Cl}$} \\
\hline $\mathrm{Na}-\mathrm{Cl}$ & 57 & 14 & 1168 & 9884 \\
\hline $\mathrm{Ca}-\mathrm{Na}-\mathrm{Cl}$ & 2 & 2 & 2122 & 2649 \\
\hline $\mathrm{Na}-\mathrm{Ca}-\mathrm{Cl}$ & 10 & 6 & 2579 & 9995 \\
\hline $\mathrm{Na}-\mathrm{Cl}-\mathrm{HCO}_{3}$ & 28 & 2 & 1081 & 6558 \\
\hline $\mathrm{Ca}-\mathrm{Na}-\mathrm{Cl}-\mathrm{HCO}_{3}$ & 2 & 2 & 1027 & 1317 \\
\hline $\mathrm{Na}-\mathrm{Ca}-\mathrm{Cl}-\mathrm{HCO}_{3}$ & 20 & 10 & 1095 & 4590 \\
\hline $\mathrm{Na}-\mathrm{Cl}-\mathrm{HCO}_{3}-\mathrm{SO}_{4}$ & 1 & 1 & 7412 & \\
\hline $\mathrm{Ca}-\mathrm{Na}-\mathrm{Cl}-\mathrm{HCO}_{3}-\mathrm{SO}_{4}$ & 2 & 2 & 1687 & 6461 \\
\hline $\mathrm{Na}-\mathrm{Ca}-\mathrm{Cl}-\mathrm{HCO}_{3}-\mathrm{SO}_{4}$ & 2 & 2 & 1631 & 7138 \\
\hline $\mathrm{Na}-\mathrm{Cl}-\mathrm{SO}_{4}-\mathrm{HCO}_{3}$ & 1 & - & 3013 & \\
\hline $\mathrm{Ca}-\mathrm{Na}-\mathrm{Cl}-\mathrm{SO}_{4}-\mathrm{HCO}{ }_{3}$ & 3 & 2 & 1821 & 3290 \\
\hline $\mathrm{Na}-\mathrm{Ca}-\mathrm{Cl}-\mathrm{SO}_{4}-\mathrm{HCO}$ & 5 & 5 & 4619 & 6111 \\
\hline $\mathrm{Na}-\mathrm{Cl}-\mathrm{SO}_{4}$ & 7 & 3 & 2028 & 9463 \\
\hline $\mathrm{Ca}-\mathrm{Na}-\mathrm{Cl}-\mathrm{SO}_{4}$ & 2 & - & 4203 & 5868 \\
\hline $\mathrm{Na}-\mathrm{Ca}-\mathrm{Cl}-\mathrm{SO}_{4}$ & 15 & 1 & 2489 & 9716 \\
\hline
\end{tabular}


Il s'agit d'eaux faiblement minéralisées ou d'eaux douces.

Leur intérêt comme eau minérale vient souvent de leur teneur en éléments spécifiques (voir fig. 3).

Leur composition est typiquement $\mathrm{Ca}-\mathrm{Mg}-\mathrm{HCO}_{3}$, en partie Ca- $\mathrm{Mg}-\mathrm{HCO}_{3}-\mathrm{SO}_{4}$ jusqu'à Ca- $\mathrm{Mg}-\mathrm{SO}_{4}$.

Seulement quelques-unes montrent des teneurs un peu plus élevées en chlorures.

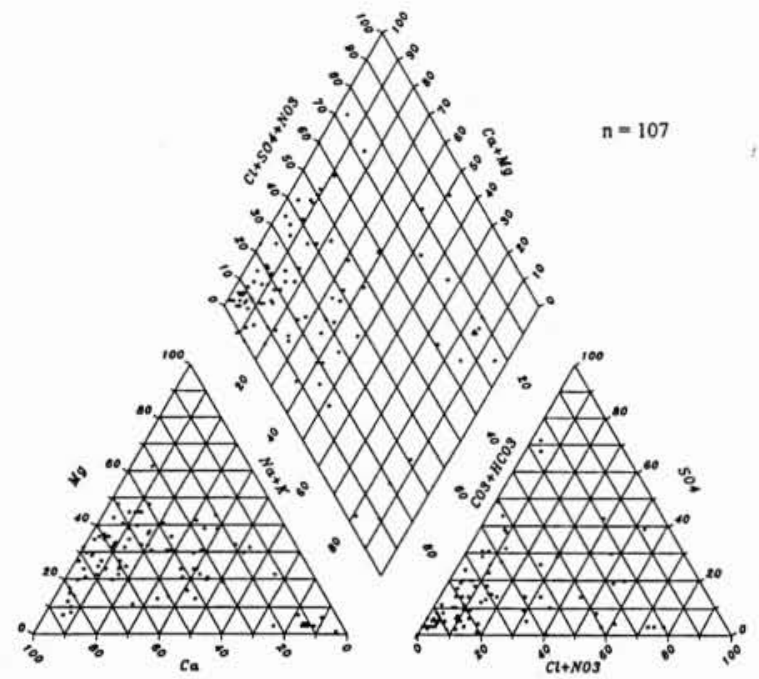

7. Diagramme PIPER pour les eaux ayant une concentration totale inférieure à $1000 \mathrm{mg} /$.

Figure 8: $1000<$ sumppm < 10000

Ici se situent la plupart des eaux minérales mises en bouteilles et utilisées dans le thermalisme.

Elles montrent une grande diversité, mais grosso modo elles peuvent être attribuées à 3 groupes :

- groupe $\mathrm{Ca}-(\mathrm{Mg}-) \mathrm{HCO}_{3}$

- groupe $\mathrm{Ca}-(\mathrm{Mg}-) \mathrm{SO}_{4}$

- groupe $\mathrm{Na}-(\mathrm{Ca}-) \mathrm{Cl}-\mathrm{SO}_{4}$.

Figure 9: sumppm > 10000

Il s'agit des eaux sodiques chlorurées, c'est-à-dire des saumures, en partie avec une teneur plus forte en $\mathrm{SO}_{4}$. Ce sont les eaux profondes qui génétiquement se trouvent toujours dans les grands bassins sédimentaires ou qui sont les résultats de la diagénèse. En outre elles prouvent également les circulations dans les systèmes souterrains régionaux.

\section{IV — LA CLASSIFICATION GÉOCHIMIQUE}

Cette classification est basée sur les règles depuis longtemps en vigueur en Allemagne. Pour la dénomination, on prend en considération les pourcentages qui dépassent 20 milli-équivalents. Ils sont d'abord rangés en ordre décroissant des cations et ensuite des anions. Le tableau 2 résume les résultats pour les eaux ayant une concentration totale (sumppm) entre 1000 et $10000 \mathrm{mg} / \mathrm{l}$.

Il existe 72 combinaisons. Les 4 groupes dominants sont les suivants :

$\mathrm{Ca}-\mathrm{Mg}-\mathrm{HCO}_{3}, \mathrm{Na}-\mathrm{HCO}_{3} \mathrm{Cl}, \mathrm{Na}-\mathrm{Cl}, \mathrm{Ca}-\mathrm{SO}_{4}$.

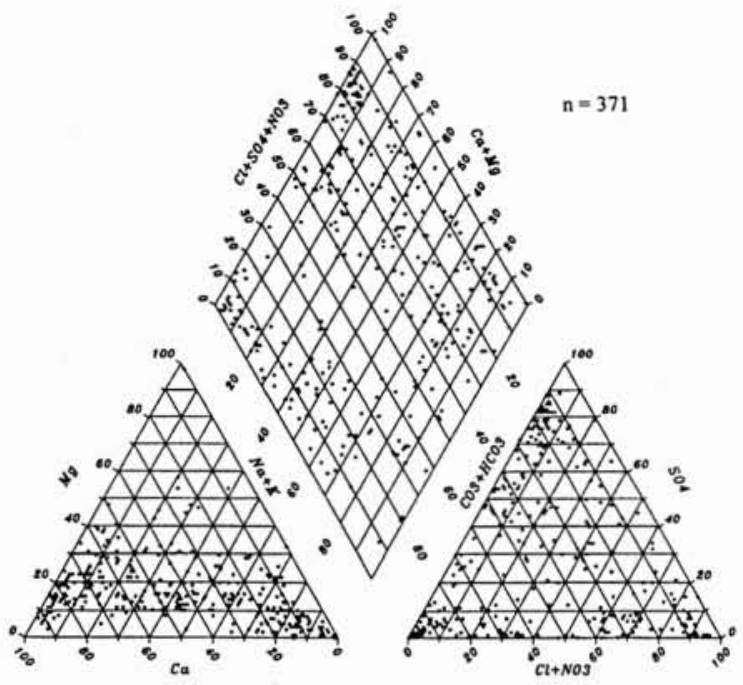

8. Diagramme PIPER pour les eaux ayant une concentration totale entre $1000 \mathrm{mg} / \mathrm{l}$ et $10000 \mathrm{mg} /$.

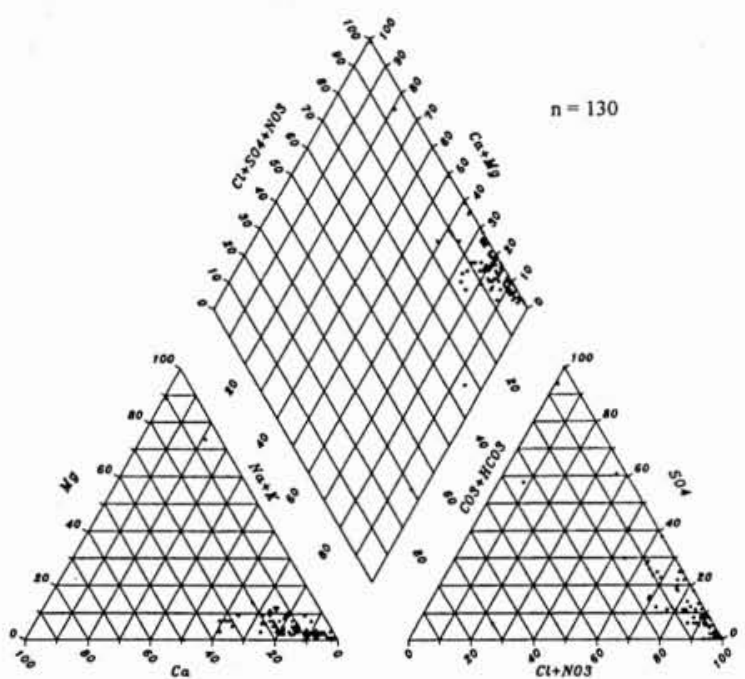

9. Diagramme PIPER pour les eaux ayant une concentration totale supérieure à $10000 \mathrm{mg} / \mathrm{l}$.

\section{Bibliographie}

CARLÉ W. (1975). - Die Mineral- und Thermalwässer von Mitteleuropa, Geologie, Chemismus, Genese. Bücher der Zeitschrift Naturwissenschaftliche Rundschau. $643 \mathrm{~S}$., 14 Abb., 1402 Analysentabellen, 15 teils zweifarbige Karten in besonderer Mappe. Stuttgart (Wissenschaftliche Verlagsanstalt).

DIENEMANN W. \& FRICKE K. (1961). - Mineral- und Heilwässer, Peloide und Heilbäder in Niedersachsen und seinen Nachbargebieten, Geologie und Lagerstätten Niedersachsens 5. Die Lagerstätten und ihre Bewirtschaftung, 5. Abt. $475 \mathrm{~S}$. Göttingen-Hannover.

FRICKE K. \& MiCHEL G. (1969). - Mineral- und Thermalwässer der Bundesrepublik Deutschland, XXIII Intern. geol. Congr. 18, 31-57. Prag. 